\title{
Challenges of Egyptian patients with systemic lupus erythematosus during the COVID-19 pandemic
}

\author{
Samar Tharwat ${ }^{1}{ }^{1 D}$, Sherin Zohdy Mohamed², Mohammed Kamal Nassar ${ }^{3}$ \\ ${ }^{1}$ Rheumatology and Immunology Unit, Department of Internal Medicine, Mansoura University, Faculty of Medicine, Egypt \\ ${ }^{2}$ Department of Internal Medicine, Horus University, Faculty of Medicine, Egypt \\ ${ }^{3}$ Mansoura Nephrology and Dialysis Unit, Department of Internal Medicine, Mansoura University, Faculty of Medicine, Egypt
}

\begin{abstract}
Objectives: The coronavirus disease 2019 (COVID-19) pandemic in Egypt is part of the worldwide COVID-19 pandemic that has contributed to substantial deterioration of healthcare systems. The aim of this study was to assess the challenges faced by Egyptian systemic lupus erythematosus (SLE) patients during the COVID-19 pandemic.

Material and methods: This questionnaire-based study was carried out on 200 patients with SLE from Egypt. The questionnaire provided covered socioeconomic status, lupus disease data, information about COVID-19 infection, and medical and family history of COVID-19 infection.

Results: The mean age of the participants was $30.1 \pm 8.4$ years. 140/200 (70\%) of the participants reported difficulty in obtaining medications during the COVID-19 pandemic, particularly antimalarials (60\%). The lupus disease condition became worse because of the drug shortage in half of the participants. Wearing protective masks (74\%) and using disinfectants of the hands several times per day (67\%) were the most reported used measures. Forty patients (20\%) had to stop or reduce taking nonsteroidal anti-inflammatory drugs while 10 patients (5\%) had to start taking antimalarials as a prophylaxis against COVID-19 infection. Among those who needed hospitalization, the main cause was lupus activity, and most of them (71\%) experienced difficulty in hospital admission. Thirty-two patients (16\%) had confirmed COVID-19 infection. About half of them had lupus flare and had to change the medications used for treatment of lupus.

Conclusions: The current COVID-19 pandemic has a negative impact on the healthcare provided to SLE patients in Egypt. Patients with SLE faced a shortage of their medications, especially antimalarials, and difficulty in hospital admission.
\end{abstract}

Key words: systemic lupus erythematosus, COVID-19, healthcare.

\section{Introduction}

The COVID-19 infection has rapidly escalated into a global health problem which started in 2019 in China and continues to spread in most countries despite control measures [1]. The COVID-19 pandemic has put an unexpected burden on healthcare systems and even threatens to overwhelm them [2].

The problem of non-COVID patients is more complex in developing countries where most patients are dependent on government hospitals [2]. In an earlier report,
Egypt had the highest risk of COVID-19 infection importation among African countries [3].

Patients with autoimmune diseases are at a higher risk of COVID-19 infection [4]. Additionally, there are many challenges faced by these patients during the COVID-19 pandemic [5]. Systemic lupus erythematosus (SLE) is a chronic autoimmune disease characterized by aberrant activity of the immune system with multiple clinical manifestations [6].

Infection is one of the most common causes of morbidity and mortality in SLE [7]. High dose glucocor- 
ticosteroids (GCs) and immunosuppressive agents are well-known risk factors for infections in addition to impaired cellular and humoral immunity among these patients [7].

To the best of our knowledge, no previous studies have evaluated the effect of the COVID-19 pandemic on Egyptian SLE patients' health status from the standpoint of the patients. The aim of this study was to assess the tremendous challenges faced by Egyptian SLE patients during the COVID-19 pandemic in terms of drug shortage, hospitalization, and COVID-19 infection.

\section{Material and methods}

This cross-sectional study was carried out on 200 SLE patients aged $>18$ years from Egypt. The study was conducted between June 2020 and December 2020. The patients were recruited from our rheumatology and immunology unit (inpatient and outpatient). Patients who had any other rheumatic, neurological or musculoskeletal diseases were excluded from the start.

The study protocol was approved by the Institutional Research Board of the Faculty of Medicine, Mansoura University (approval registration number: R.21.03.1257). The study was explained to all participants and informed written consent was obtained from each one of them before starting the study. Sociodemographic, clinical and therapeutic data were collected.

The researchers distributed the questionnaire among participants. The questionnaire was administered by 2 methods: either self-administered for those who could read and write or researcher-administered for those who could not. The questionnaire was originally designed to answer questions about health status, knowledge and information about the COVID-19 pandemic and medical and family history of the COVID-19 infection in rheumatoid arthritis (RA) patients [8].

All items had the same contents with the wording revised to reflect SLE instead of RA. A "yes" or "no" response was provided and all questions were closed (only 2 questions in the "History of the COVID-19 infection" section were open-ended).

\section{Statistical analysis}

Statistical analysis was performed with SPSS 20.0 for Windows (SPSS Inc., Chicago, IL, USA). Continuous data were expressed as mean \pm standard deviation or percentages and frequency; the Shapiro-Wilk test was used to assess the normality of distribution.

\section{Results}

The sample consisted of 200 SLE patients. The patients' data are described in Table I.
The mean age of the participants was 30.1 years (with SD \pm 8.4 ). Among the participants, 180 (90\%) were female and 172 (86\%) were from urban areas. About three quarters of the studied patients were highly educated and $80 \%$ belonged to the medium economic class.

Lupus activity scores showed inactivity in 65\%, mild in $17 \%$, moderate in $16 \%$ and severe disease activity in $3 \%$ of the study patients. Corticosteroids and mycophenolate mofetil (MMF) and antimalarials were the most used medications ( $87 \%, 87 \%$ and $70 \%$ respectively).

Most patients (90\%) were compliant with therapy. However, 140/200 (70\%) of the participants reported difficulty in obtaining medications during the COVID-19 pandemic. It was particularly hard to obtain antimalarials (60\%). Lupus disease activity was affected by the drug shortage in about half of the study participants.

The protective measures taken for COVID-19 infection, sources of information about the COVID-19 virus and the impact of the pandemic on the participants are shown in Table II. Twenty-eight (14\%) patients reported that they had lost their jobs while 26 patients (13\%) reported that their work was severely impacted by the crisis.

Among the precautions taken for prophylaxis against infection, wearing protective masks (74\%) and using disinfectants of the hands several times per day (67\%) were the most reported measures.

Forty patients (20\%) had to stop or reduce taking NSAIDs to guard against COVID-19 infection while 10 patients (5\%) had to start taking antimalarials as a prophylaxis against COVID-19 infection.

Among 34 patients (17\%) who needed hospitalization, 26 (76\%) were mainly due to lupus activity and 24 (71\%) of them experienced difficulty in hospital admission because of the COVID-19 pandemic.

About $40 \%$ of the participants reported that they had at least one close relative with confirmed COVID-19 infection. In addition, thirty-two patients (16\%) had confirmed COVID-19 infection. Most of them became clinically free after treatment.

However, about half of them had lupus flare and had to change the medications used for treatment of SLE. Cutaneous and musculoskeletal manifestations were the most frequently reported presentation of SLE flare following COVID-19 infection. Figure 1 illustrates the disparities in questionnaire responses between males and females.

Among studied patients $58.6 \%$ of females vs. $20 \%$ of males would use telemedicine for remote contact with the rheumatologist. Most female patients were keener than males to wear masks (76.7\% vs. 50\% respectively).

Figure 2 illustrates the difference in response to the questionnaire between the patients from urban and rural areas. 
Table I. Sociodemographic and health status of the patients including the medications received, availability and their contact with the rheumatologist

\begin{tabular}{|c|c|}
\hline Parameters & $\begin{array}{c}\text { SLE patients }(n=200) \\
n(\%)\end{array}$ \\
\hline \multicolumn{2}{|c|}{ Sociodemographic parameters } \\
\hline Age [years] & $30.1 \pm 8.4$ \\
\hline Gender (female) & $180(90)$ \\
\hline \multicolumn{2}{|l|}{ Residence } \\
\hline Urban & $172(86)$ \\
\hline Rural & $28(14)$ \\
\hline \multicolumn{2}{|l|}{ Education level } \\
\hline Low & $14(7)$ \\
\hline Moderate & $38(19)$ \\
\hline High & $148(74)$ \\
\hline \multicolumn{2}{|l|}{ Economic class } \\
\hline Low & $18(9)$ \\
\hline Medium & $160(80)$ \\
\hline High & $22(11)$ \\
\hline \multicolumn{2}{|l|}{ Special habits } \\
\hline Tea/coffee & $120(60)$ \\
\hline Smoking & $16(8)$ \\
\hline Alcohol & $4(2)$ \\
\hline Addiction & $2(1)$ \\
\hline \multicolumn{2}{|l|}{ Lupus activity (SLEDAI) } \\
\hline Inactive & $130(65)$ \\
\hline Mild & $34(17)$ \\
\hline Moderate & $31(16)$ \\
\hline Severe & $5(3)$ \\
\hline \multicolumn{2}{|c|}{ Health status during COVID-19 pandemic parameters } \\
\hline \multicolumn{2}{|l|}{ Medications used } \\
\hline Corticosteroids & $174(87)$ \\
\hline $\mathrm{CQ} / \mathrm{HCQ}$ & $140(70)$ \\
\hline Azathioprine & $10(5)$ \\
\hline Cyclosporine & $62(31)$ \\
\hline Mycophenolate mofetil & $174(87)$ \\
\hline
\end{tabular}

\begin{tabular}{|c|c|}
\hline Parameters & $\begin{array}{c}\text { SLE patients }(n=200) \\
n(\%)\end{array}$ \\
\hline Methotrexate & $2(1)$ \\
\hline Leflunomide & $6(3)$ \\
\hline Intravenous immunoglobulin & $4(2)$ \\
\hline Rituximab & $4(2)$ \\
\hline \multicolumn{2}{|l|}{ Mode of obtaining medications } \\
\hline Self-payment & $152(76)$ \\
\hline Medical insurance & $42(21)$ \\
\hline Governmental supply & $12(6)$ \\
\hline Regularity of drug intake & $180(90)$ \\
\hline Difficulty to obtain the drug & $140(70)$ \\
\hline Corticosteroids & $8(4)$ \\
\hline Azathioprine & $12(6)$ \\
\hline Mycophenolate mofetil & $8(4)$ \\
\hline $\mathrm{CQ} / \mathrm{HCQ}$ & $120(60)$ \\
\hline Disease affected by drug shortage & $96(48)$ \\
\hline $\begin{array}{l}\text { Regular contact with } \\
\text { the rheumatologist }\end{array}$ & $174(87)$ \\
\hline Modes of remote contact & $110(55)$ \\
\hline Phone & $76(38)$ \\
\hline WhatsApp & $94(47)$ \\
\hline Messenger & $24(12)$ \\
\hline Telegram & $2(1)$ \\
\hline Physician's website/page & $6(3)$ \\
\hline \multicolumn{2}{|l|}{ Associated diseases/comorbidities } \\
\hline Diabetes mellitus & $4(2)$ \\
\hline Hypertension & $26(13)$ \\
\hline Cardiovascular & $6(3)$ \\
\hline Chest problems & $12(6)$ \\
\hline Renal problems & $22(11)$ \\
\hline Hepatic problems & $2(1)$ \\
\hline Allergy problems & $10(5)$ \\
\hline
\end{tabular}

CQ/HCQ - hydroxychloroquine, SLE - systemic lupus erythematosus, SLEDAI - Systemic Lupus Disease Activity Index. All parameters are described as number (percent), except age, which is described as mean $\pm S D$.

\section{Discussion}

Systemic lupus erythematosus is a chronic autoimmune multisystem disorder with a broad-spectrum clinical presentation. The incidence and prevalence of SLE are significantly increasing worldwide [9].

Not much is known about the impact of the COVID-19 pandemic on SLE patients. In this questionnaire-based study, we studied the tremendous challenges faced by
Egyptian SLE patients during the COVID-19 pandemic in terms of drug shortage, hospitalization, and COVID-19 infection.

A significant number of our participants $n=140$ (70\%) reported difficulty to obtain drugs during the COVID-19 pandemic. Medication shortages were of particular concern during the COVID-19 outbreak [10]. In the same context, antimalarial drug shortage due to the COVID-19 outbreak affected more than half of our study cohort (60\%). 
Table II. Protective measures taken for COVID-19, source of information and influence of the pandemic

\begin{tabular}{|c|c|}
\hline Parameters (during COVID-19 pandemic) & $\begin{array}{c}\text { SLE patients } \\
(n=200) \\
n(\%)\end{array}$ \\
\hline \multicolumn{2}{|l|}{ Work affected } \\
\hline Mild & $54(27)$ \\
\hline Moderate & $38(19)$ \\
\hline Severe & $26(13)$ \\
\hline Lost & $28(14)$ \\
\hline \multicolumn{2}{|l|}{ Protective measures taken } \\
\hline Staying at home & $116(58)$ \\
\hline Wearing a mask & $148(74)$ \\
\hline Social distancing & $122(62)$ \\
\hline Using disinfectants/alcohol & $134(67)$ \\
\hline \multicolumn{2}{|l|}{ Source of information on COVID-19 } \\
\hline Physicians & $56(28)$ \\
\hline Friends & $26(13)$ \\
\hline Media & $116(58)$ \\
\hline Social media & $150(75)$ \\
\hline Others (relatives/MOH/at work) & $88(44)$ \\
\hline Consider their knowledge sufficient & $120(60)$ \\
\hline Stopped or reduced taking NSAIDs & $40(20)$ \\
\hline Considered complementary therapy & $46(23)$ \\
\hline Received hydroxychloroquine for prevention & $10(5)$ \\
\hline Needed hospitalization & $34(17)$ \\
\hline Activity & $26(13)$ \\
\hline Pulmonary embolism & $6(3)$ \\
\hline Premature labor & $2(1)$ \\
\hline Found difficulty in hospitalization & $24(12)$ \\
\hline \multicolumn{2}{|l|}{ History of COVID-19 infection } \\
\hline Close relatives affected & $76(38)$ \\
\hline Protective measures considered & $68(34)$ \\
\hline Patients with confirmed COVID-19 & $32(16)$ \\
\hline Free after treatment & $24(12)$ \\
\hline Carrier & $2(1)$ \\
\hline Still infected & $6(3)$ \\
\hline The disease was affected (flare) & $18(9)$ \\
\hline Cutaneous manifestations & 7 \\
\hline Arthritis & 5 \\
\hline Lupus nephritis & 2 \\
\hline Livedoid vasculopathy & 1 \\
\hline Lupus vasculitis & 1 \\
\hline Unknown & 2 \\
\hline Change in the lupus medications & $16(8)$ \\
\hline
\end{tabular}

$\mathrm{MOH}$ - Ministry of Health, NSAIDs - nonsteroidal anti-inflammatory drugs.
Antimalarials are commonly used as the standard of care for management of SLE [11]. The use of antimalarial agents in SLE is very beneficial [12]. Their use is associated with lower rates of new organ damage, disease activity and thromboembolic events [13].

Also, antimalarials have recently been touted as one of the weapons against COVID-19 infection [14]. Additionally, US President Donald Trump stated that antimalarials showed great promise and "could be a gamechanger" in the treatment of COVID-19 infection [15].

However, a retrospective multicenter study published in the Journal of the American Medical Association [16] showed that use of hydroxychloroquine (HCQ) to treat COVID-19 infected patients was associated with higher mortality.

Nevertheless, there is widespread off-label use of antimalarials for both prophylaxis and treatment of COVID-19 infection, especially in African countries [17], which may be the main cause of shortage of these drugs [18].

In a national survey conducted on 531 Canadian rheumatologists, about three quarters of the responders were concerned about the antimalarial drug shortage, while $60 \%$ of them had been contacted by the patients regarding difficulties accessing HCQ [19].

Shortage of these drugs may cause significant problems for SLE patients who are currently being treated with these drugs [20]. In a retrospective chart review of 509 SLE patients, patients who discontinue antimalarials were at higher risk of disease flares [21].

Unfortunately, no other drug provides similar benefits as HCQ [22]. Therefore, SLE patients are facing challenges with the improper use of their essential medications, and health care policies should take this point into account [23].

To solve this problem in Italy, SLE patients are advised to reduce their doses of antimalarial agents in order to overcome the widespread shortage of these medications [15]. In this context, the German Federal Institute for Drugs and Medical Devices reiterates that any prescription of HCQ in Germany should include an in-label diagnosis justifying its use and prohibits off-label use of that drug except for clinical trials [24].

Our results showed that patients who reported drug shortage also reported more exacerbation of lupus manifestations. It is well known that stoppage of medications of SLE is associated with disease flare [25].

Systemic lupus erythematosus patients have been overwhelmed with warnings of their higher risk of COVID-19 infection and severe illness [15]. Shortage of essential medications for SLE patients may be associated with high emotional distress and anxiety, which may also be associated with a worse disease outcome [26]. 


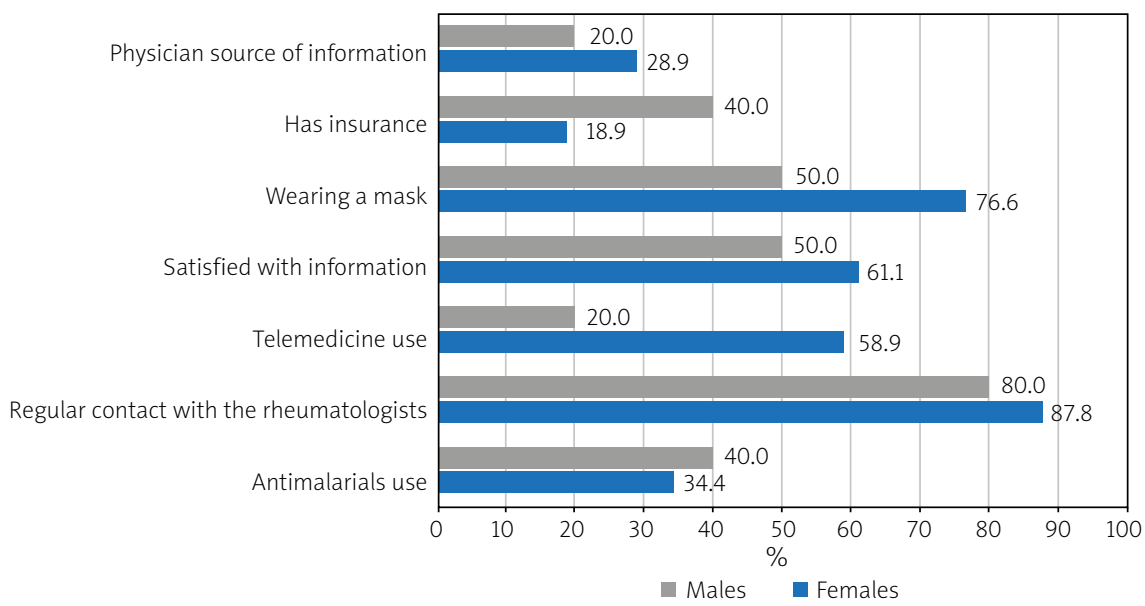

Fig. 1. Frequency of responses to questionnaire in Egyptian lupus patients by gender.

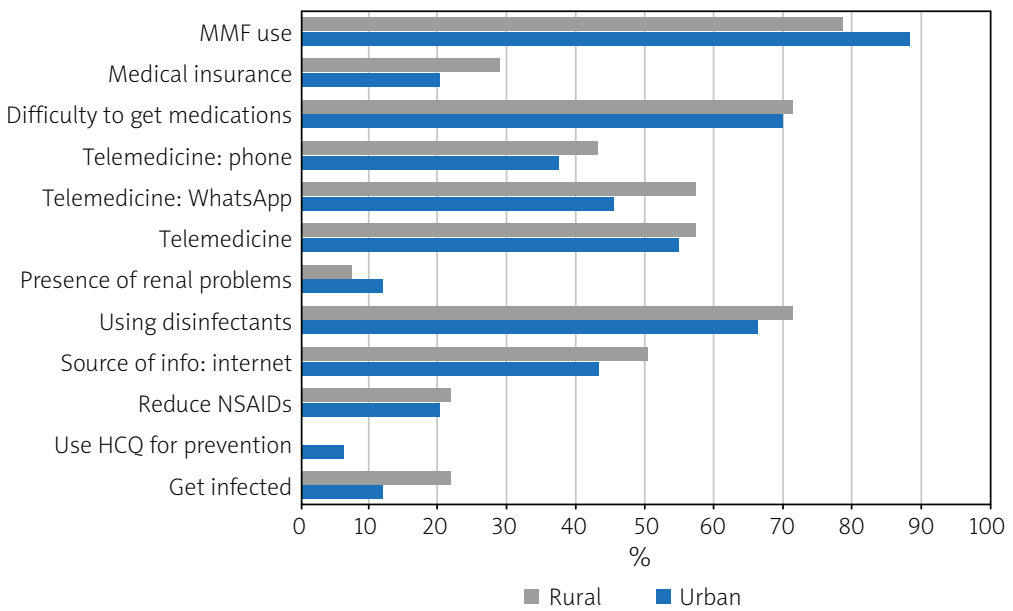

HCQ - hydroxychloroquine, MMF - mycophenolate mofetil, NSAIDs - nonsteroidal anti-inflammatory drugs.

Fig. 2. Frequency of responses to questionnaire in Egyptian lupus patients by residence.

Most of the study patients stated that they were in regular contact with the rheumatologist during the COVID-19 pandemic. In point of fact, SLE patients face challenges in disease followup during the COVID-19 pandemic. Those who keep visiting the doctor are at risk of COVID-19 infection, while at the same time, others are at risk of disease flare [27].

Therefore, it may be better to replace physical consultations of patients with SLE by telemedicine consultations in order to prevent the risk of contagion [28]. In Germany for example, the COVID-19 pandemic led to acute restriction of outpatient care options for patients with rheumatic diseases, which led to a temporary reduction of patients' visits to doctors' offices by about 50\% [29]. As a result, medical care was widely switched to telemedicine [29].

A substantial number of the participants used telemedicine as a mode of contact with their physician. In a survey conducted among members of the Dutch Rheumatology Society, the results revealed that more than $80 \%$ of outpatients' visits were performed via telephone during the pandemic [30]. However, it is not easy to judge which patients may be suitable for telemedicine consultation [31].

A significant proportion of our cohort (17\%) reported that they were in need of hospitalization during the COVID-19 pandemic. The most common reason for hospitalization was lupus flare. COVID-19 related distress in rheumatic patients is associated with worse health scores [32]. Disease flare and medication access difficulties were common among rheumatic patients during the COVID-19 pandemic [32].

Regrettably, most of those who needed hospitalization experienced difficulty in hospital admission. The COVID-19 pandemic is associated with "collateral damage" caused by the decrease of emergency room visits for non-COVID-19 conditions [33].

There is a decrease (65.4\%) in hospitalization of SLE patients during the COVID-19 pandemic [34]. The pan- 
demic has promoted crowding of hospital places with COVID-19 patients. Some hospitals had to suspend other services during the pandemic [35].

Systemic lupus erythematosus patients are at increased risk of infection either due to lupus itself or immunosuppressive medications [36]. The initial results of the COVID-19 Global Rheumatology Alliance Registry show that 19 (17\%) of 110 rheumatic patients diagnosed with COVID-19 were patients with lupus [37].

According to our results, $16 \%$ of the studied patients reported confirmed infection with COVID-19. The clinical course of our infected cohort seems to be favorable, as most of them (75\%) reported complete resolution of infection without significant complications. This is compatible with the results of a survey conducted among 126 lupus patients in Italy which revealed 12 patients with confirmed or suspected COVID-19 infection [38].

The most recent studies showed controversial results about COVID-19 sequelae in SLE patients [39]. It seems that SLE patients may be at higher risk of an unfavorable outcome during the COVID-19 pandemic [40].

\section{Conclusions}

This study had some limitations. The method of data collection was based on self-reporting. The information provided by the participants could not be verified for clinical accuracy. Additionally, we could not get information from patients with severe COVID-19 infection. The small number of the participants may be another limitation. Finally, the COVID-19 outbreak is still ongoing, and possible new infections in our cohort cannot be excluded.

However, this study had several strengths; it targeted an Egyptian cohort. To the best of our knowledge, this is the first paper that addresses the impact of the COVID-19 pandemic in Egyptian lupus patients.

Further studies on a large number of patients and from different centers in Egypt are warranted to confirm that these data help improve management of SLE patients during the COVID-19 pandemic. Multinational studies may also shed light on this topic.

The authors declare no conflict of interest.

\section{References}

1. World Health Organization. WHO Coronavirus (COVID-19) Dashboard, https://covid19.who.int/ [access: 8.04.2021].

2. Rathi M, Singh P, Bi HP, et al. Impact of the COVID-19 pandemic on patients with systemic lupus erythematosus: observations from an Indian inception cohort. Lupus 2021; 30: 158-164, DOI: 10.1177/0961203320962855.

3. Gilbert M, Pullano G, Pinotti F, et al. Preparedness and vulnerability of African countries against importations of COVID-19: a modelling study. Lancet 2020; 395: 871-877, DOI: 10.1016/ S0140-6736(20)30411-6.

4. Murtas R, Andreano A, Gervasi F, et al. Association between autoimmune diseases and COVID-19 as assessed in both a test-negative case-control and population case-control design. Auto Immun Highlights 2020; 11: 15, DOI: 10.1186/s13317020-00141-1.

5. Talarico R, Aguilera S, Alexander $T$, et al. The impact of COVID-19 on rare and complex connective tissue diseases: the experience of ERN ReCONNET. Nat Rev Rheumatol 2021; 17: 177-184, DOI: 10.1038/s41584-020-00565-z.

6. Aringer M, Costenbader K, Daikh D, et al. 2019 European League Against Rheumatism/American College of Rheumatology classification criteria for systemic lupus erythematosus. Arthritis Rheumatol 2019; 71: 1400-1412, DOI: 10.1002/ art.40930.

7. Barber MRW, Clarke AE. Systemic lupus erythematosus and risk of infection. Expert Rev Clin Immunol 2020; 16: 527-538, DOI: 10.1080/1744666X.2020.1763793.

8. Abualfadl E, Ismail F, Shereef RRE, et al. Impact of COVID-19 pandemic on rheumatoid arthritis from a Multi-Centre patient-reported questionnaire survey: influence of gender, ruralurban gap and north-south gradient. Rheumatol Int 2021; 41: 345-353, DOI: 10.1007/s00296-020-04736-9.

9. Fatoye F, Gebrye T, Svenson LW. Real-world incidence and prevalence of systemic lupus erythematosus in Alberta, Canada. Rheumatol Int 2018; 38: 1721-1726, DOI: 10.1007/s00296-0184091-4.

10. Shuman AG, Fox ER, Unguru Y. COVID-19 and drug shortages: a call to action. J Manag Care Spec Pharm 2020; 26: 945-947, DOI: $10.18553 /$ jmcp.2020.26.8.945.

11. Hassan F, Naffaa ME, Paz Z. Value of antimalarial drugs in the treatment of lupus. Systemic Lupus Erythematosus. $2^{\text {nd }}$ ed. Elsevier, Amsterdam 2021: 591-595.

12. Gomez A, Soukka S, Johansson P, et al. Use of antimalarial agents is associated with favourable physical functioning in patients with systemic lupus erythematosus. J Clin Med 2020; 9: 1813, DOI: 10.3390/jcm9061813.

13. Fessler BJ, Alarcón GS, McGwin G Jr, et al. Systemic lupus erythematosus in three ethnic groups: XVI. Association of hydroxychloroquine use with reduced risk of damage accrual. Arthritis Rheum 2005; 52: 1473-1480, DOI: 10.1002/art.21039.

14. Schrezenmeier EV, Burmester GR, Eckardt KU, Dörner T. Role for antimalarials in the management of COVID-19. Curr Opin Rheumatol 2020; 32: 449-457, DOI: 10.1097/BOR. 0000000000000731.

15. Peschken CA. Possible consequences of a shortage of hydroxychloroquine for patients with systemic lupus erythematosus amid the COVID-19 pandemic. J Rheumatol 2020; 47: 787-790, DOI: 10.3899/jrheum.200395.

16. Rosenberg ES, Dufort EM, Udo T, et al. Association of treatment with hydroxychloroquine or azithromycin with in-hospital mortality in patients with COVID-19 in New York State. JAMA 2020; 323: 2493-2502, DOI: 10.1001/jama.2020.8630.

17. Belayneh A. Off-label use of chloroquine and hydroxychloroquine for COVID-19 treatment in Africa against WHO recommendation. Res Rep Trop Med 2020; 11: 61-72, DOI: 10.2147/ RRTM.S269936. 
18. Dejaco C, Alunno A, Bijlsma JW, et al. Influence of COVID-19 pandemic on decisions for the management of people with inflammatory rheumatic and musculoskeletal diseases: a survey among EULAR countries. Ann Rheum Dis 2020; annrheumdis-2020-218697, DOI: 10.1136/annrheumdis-2020-218697 [Online ahead of print].

19. Mendel A, Bernatsky S, Askanase A, et al. Hydroxychloroquine shortages during the COVID-19 pandemic. Ann Rheum Dis 2020; 80: 1-2, DOI: 10.1136/annrheumdis-2020-218164.

20. Jakhar D, Kaur I. Potential of chloroquine and hydroxychloroquine to treat COVID-19 causes fears of shortages among people with systemic lupus erythematosus. Nat Med 2020; 26: 632, DOI: 10.1038/s41591-020-0853-0.

21. Aouhab Z, Hong $\mathrm{H}$, Felicelli $\mathrm{C}$, et al. Outcomes of systemic lupus erythematosus in patients who discontinue hydroxychloroquine. ACR Open Rheumatol 2019; 1: 593-599, DOI: 10.1002/acr2.11084.

22. Husayn SS, Brown JD, Presley CL, et al. Hydroxychloroquine alternatives for chronic disease: response to a growing shortage amid the global COVID-19 pandemic. J Pharm Pract 2020 897190020942658, DOI: 10.1177/0897190020942658 [Online ahead of print].

23. Plüß M, Chehab G, Korsten P. Concerns and needs of patients with systemic lupus erythematosus regarding hydroxychloroquine supplies during the COVID-19 pandemic: results from a patient-centred survey. Ann Rheum Dis 2020; annrheumdis-2020-217967, DOI: 10.1136/annrheumdis-2020-217967 [Online ahead of print].

24. Sattui SE, Liew JW, Graef ER, et al. Swinging the pendulum: lessons learned from public discourse concerning hydroxychloroquine and COVID-19. Expert Rev Clin Immunol 2020; 16: 659-666, DOI: 10.1080/1744666X.2020.1792778.

25. Hanaoka H, lida H, Kiyokawa T, et al. Glucocorticoid, immunosuppressant, hydroxychloroquine monotherapy, or no therapy for maintenance treatment in systemic lupus erythematosus without major organ manifestations. Clin Rheumatol 2019; 38: 2785-2791, DOI: 10.1007/s10067-019-04633-y.

26. Delis PC. Uncertainty and quality of life in systemic lupus erythematosus: a cross-sectional study. Rehabil Nurs 2019; 44 2-10, DOI: 10.1097/rnj.0000000000000118.

27. Ugarte-Gil MF, Acevedo-Vásquez E, Alarcón GS, et al. The number of flares patients experience impacts on damage accrual in systemic lupus erythematosus: data from a multiethnic Latin American cohort. Ann Rheum Dis 2015; 74: 1019-1023, DOI: 10.1136/annrheumdis-2013-204620.

28. Hollander JE, Carr BG. Virtually perfect? Telemedicine for COVID-19. N Engl J Med 2020; 382: 1679-1681, DOI: 10.1056/ NEJMp2003539.

29. Schulze-Koops H, Specker C, Krueger K. Telemedicine holds many promises but needs to be developed to be accepted by patients as an alternative to a visit to the doctor. Response to: 'Patient acceptance of using telemedicine for follow-up of lupus nephritis in the COVID-19 outbreak' by So et al. Ann Rheum Dis 2020; annrheumdis-2020-218235: DOI: 10.1136/ annrheumdis-2020-218235 [Online ahead of print].

30. Bos WH, van Tubergen A, Vonkeman H. Telemedicine for patients with rheumatic and musculoskeletal diseases during the COVID-19 pandemic; a positive experience in the Nether- lands. Rheumatol Int 2021; 41: 565-573, DOI: 10.1007/s00296020-04771-6.

31. Taylor PC. Adopting PROs in virtual and outpatient management of RA. Nat Rev Rheumatol 2020; 16: 477-478, DOI: 10.1038/s41584-020-0449-6.

32. Maldonado D, Tu E, Mahmood SN, et al. Medication access difficulty and COVID-related distress are associated with disease flares in rheumatology patients during the COVID-19 pandemic. Arthritis Care Res (Hoboken) 2021; 73: 1162-1170, DOI: 10.1002/acr.24531.

33. Masroor S. Collateral damage of COVID-19 pandemic: delayed medical care. J Card Surg 2020; 35: 1345-1357, DOI: 10.1111/ jocs.14638.

34. Chuah SL, Teh CL, Wan Mohd Akbar SA, et al. Impact of COVID-19 pandemic on hospitalisation of patients with systemic lupus erythematosus (SLE): report from a tertiary hospital during the peak of the pandemic. Ann Rheum Dis 2020; annrheumdis-2020-218475, DOI: 10.1136/annrheumdis-2020218475 [Online ahead of print].

35. Fazal F, Gupta N, Khot W, Ray Y. Collateral damage due to COVID-19. Trop Doct 2021; 51: 126-127, DOI: 10.1177/0049475520942387.

36. Yuan Q, Xing X, Lu Z, Li X. Clinical characteristics and risk factors of infection in patients with systemic lupus erythematosus: a systematic review and meta-analysis of observational studies. Semin Arthritis Rheum 2020; 50: 1022-1039, DOI: 10.1016/j.semarthrit.2020.06.004.

37. Gianfrancesco MA, Hyrich KL, Gossec L, et al. Rheumatic disease and COVID-19: initial data from the COVID-19 global rheumatology alliance provider registries. Lancet Rheumatol 2020; 2: e250-e253, DOI: 10.1016/S2665-9913(20)30095-3.

38. Bozzalla Cassione E, Zanframundo G, Biglia A, et al. COVID-19 infection in a northern-Italian cohort of systemic lupus erythematosus assessed by telemedicine. Ann Rheum Dis 2020; 79: 1382-1383, DOI: 10.1136/annrheumdis-2020-217717.

39. Favalli EG, Agape E, Caporali R. Are patients with systemic lupus erythematosus at increased risk of severe COVID-19? J Rheumatol 2020; 47: 1592, DOI: 10.3899/jrheum.200723.

40. Favalli EG, Gerosa M, Murgo A, Caporali R. Are patients with systemic lupus erythematosus at increased risk for COVID-19? Ann Rheum Dis 2021; 80: e25, DOI: 10.1136/annrheumdis2020-217787. 